\title{
Вмј Global Health How the health-seeking behaviour of pregnant women affects neonatal outcomes: findings of system dynamics modelling in Pakistan
}

\author{
Raheelah Ahmad, ${ }^{1,2}$ Nina Jiayue Zhu, ${ }^{1}$ Reda Mohamed Lebcir, ${ }^{3}$ Rifat Atun ${ }^{4}$
}

To cite: Ahmad R, Zhu NJ, Lebcir RM, et al. How the health-seeking behaviour of pregnant women affects neonatal outcomes: findings of system dynamics modelling in Pakistan. BMJ Glob Health 2019:4:e001242. doi:10.1136/ bmjgh-2018-001242

Handling editor Dr Sanni Yaya

- Additional material is published online only. To view please visit the journal online (http://dx.doi.org/10.1136/ bmjgh-2018-001242).

RA and NJZ contributed equally.

Received 19 0ctober 2018 Revised 25 January 2019 Accepted 1 February 2019

Check for updates

(c) Author(s) (or their employer(s)) 2019. Re-use permitted under CC BY-NC. No commercial re-use. See rights and permissions. Published by BMJ.

${ }^{1}$ NIHR Health Protection Research Unit in Healthcare Associated Infection and Antimicrobial Resistance, Imperial College London, London, UK

${ }^{2}$ Institute of Business

Administration, Karachi, Karachi, Pakistan

${ }^{3}$ University of Hertfordshire Business School, Hatfield, UK

${ }^{4}$ School of Public Health, Harvard University, Boston, Massachusetts, USA

Correspondence to Dr Rifat Atun;

ratun@hsph.harvard.edu

\section{ABSTRACT}

Background Limited studies have explored how healthseeking behaviour during pregnancy through to delivery affect neonatal outcomes. We modelled health-seeking behaviour across urban and rural settings in Pakistan, where poor neonatal outcomes persist with wide disparities.

Methods and findings A system dynamics model was developed and parameterised. Following validation tests, the model was used to determine neonatal mortality for pregnant women considering their decisions to access, refuse and switch antenatal care services in four provider sectors: public, private, traditional and charitable. Four health-seeking scenarios were tested across different pregnancy trimesters. Health-seeking behaviour in different subgroups by geographical locations and social network effect was modelled. The largest reduction in neonatal mortality was achieved with antenatal care provided by skilled providers in public, private or charitable sectors, combined with the use of institutional delivery. Women's social networks had strong influences on if, when and where to seek care. Interventions by Lady Health Workers had a minimal impact on health-seeking behaviour and neonatal outcomes after trimester 1. Optimal benefits were achieved for urban women when antenatal care was accessed within trimester 2 , but for rural women within trimester 1. Antenatal care access delayed to trimester 3 had no protective impact on neonatal mortality. Conclusions System dynamics modelling enables capturing the complexity of health-seeking behaviours and impact on outcomes, informing intervention design, implementation of targeted policies and uptake of services specific to urban/rural settings considering structural enablers/barriers to access, cultural contexts and strong social network influences.

\section{INTRODUCTION}

Since 1990, maternal mortality has declined worldwide by $50 \%$, but this decline has been uneven among and within countries. According to WHO estimates in 2015, 830 women died each day from preventable causes associated with pregnancy and childbirth, and 3.1 million newborns died in their

\section{Key questions}

What is already known?

- Pakistan remains among the poorest performer worldwide on neonatal survival.

- Structural, cultural, individual socioeconomic factors and social networks collectively influence health-seeking behaviour of women during pregnancy.

What are the new findings?

- Interventions by Lady Health Workers after trimester 1 had minimal impact on health-seeking behaviou and neonatal outcomes.

- Optimal benefits were achieved for urban women when antenatal care (ANC) was accessed within trimester 2, but for rural women within trimester 1 ANC access delayed to trimester 3 had no protective impact on neonatal mortality.

What do the new findings imply?

- The model identifies the optimal time, mix and scale of interventions health systems can employ for different population groups (eg, rural and urban, different socioeconomic groups) to improve neonatal outcomes.

first month of life. Almost $99 \%$ of all maternal deaths occurred in low-income and middle-income countries (LMICs). ${ }^{1}$ More than half of the global neonatal deaths in 2009 occurred in five countries including Pakistan (India, Nigeria, China and the Democratic Republic of Congo), ${ }^{2}$ and Pakistan remains the 11 th worst performing country in the world on this indicator. $^{3}$

WHO guidelines, in 2016, increased the recommended number of contacts between the mother and healthcare providers at time points that may facilitate assessment of wellbeing and provision of interventions from four to eight antenatal care (ANC) appointments along with skilled attendance at birth, ${ }^{4}$ to reduce maternal and neonatal mortality 
(deaths in 0-27 days per 1000 live births). Yet, worldwide, even this minimum uptake is achieved by only two-thirds of pregnant women. ${ }^{5}$ Community-based interventions such as home visiting and counselling can help to reduce neonatal mortality in LMICs. ${ }^{6-8}$ But availability of ANC services does not assure uptake, ${ }^{9}$ and an understanding of health-seeking behaviour during pregnancy is critical to ensure effective ANC uptake.

In Pakistan, the average ANC coverage is $37 \%$ and skilled attendance at birth 52\%, but ANC coverage ranges from $29.8 \%$ to $85.2 \%$ among different socioeconomic groups, ${ }^{10}$ influenced by the supply of services and demand for them. ${ }^{511}$

Pakistan's Lady Health Worker (LHW) programme initiated in 1994 aimed to improve maternal and child health outcomes by deploying locally recruited female health workers to provide community-based primary healthcare. The programme has helped to reduce infant mortality due to higher immunisation uptake, ${ }^{1}$ but with limited improvements in ANC uptake, ${ }^{9}{ }^{10}$ with implications for neonatal mortality.

The pluralistic health system for ANC comprises traditional care from healers and untrained dais (traditional birth attendants), and professional services from public-funded facilities (hospital, rural health centres, basic health units), privately funded and privately-run facilities (hospitals, single practitioner clinics), or charity-funded hospitals. There is no formal gatekeeper enforced, resulting in direct access across, and within, these provider domains. ${ }^{12}$

Women in Pakistan experience social vulnerability and disempowerment, with poor nutrition, limited education, early marriage and high fertility rates, and the LHW programme was designed to address these factors that adversely affect women's health-seeking behaviour. ${ }^{13} 14$ While qualitative studies in Pakistan have identified structural and cultural factors that influence women's healthseeking behaviour, they did not examine how these barriers could be overcome in order to improve maternal and neonatal outcomes. ${ }^{51516}$

To date, no studies have explored how health-seeking behaviours along the continuum of care during pregnancy through to delivery affect neonatal outcomes, especially in countries such as Pakistan which have poor neonatal outcomes with large disparities. ${ }^{16} 17$ In this study, we use mixed-methods research to trace the ANC journey of women in Pakistan during three trimesters to explore factors influencing their health-seeking behaviour.

\section{METHODS}

We use mixed-methods research to explore the effects of health-seeking behaviour of women during pregnancy on neonatal mortality. The factors influencing health-seeking were elicited from (1) the literature, (2) a qualitative cohort study of 60 women (online supplementary material 2) and (3) the Pakistan Demographic and Health Survey 2017-2018. ${ }^{18}$ Using system dynamics (SD) modelling, we modelled how different timing and access to care during pregnancy impact neonatal outcomes.

SD modelling is a powerful analytical tool used for analysing complex health system management issues to improve system performance or shape better healthcare provision. ${ }^{19-22} \mathrm{SD}$ modelling is most useful when analysing complex systems with cause-effect relationships between key variables where reinforcing or negative feedback relations exist between these variables. SD modelling allows for simulation of outcome indicators of interest when we change values of the variables, quantitatively, using computer-based software programs. ${ }^{19} 20$ The method has demonstrated its capability in solving health system management problems when employed to simulate population flows, predict health-seeking behaviour, support health decision-making, test health policy alternatives and evaluate effectiveness of health interventions. ${ }^{23-26}$ Specifically, SD modelling has been applied to model behaviours, to assess the impact of educational interventions on the abuse of pharmaceutical opioids in the USA. The approach has been useful for assessing structural and process measures for the management of waiting times for patients with coronary heart disease in the UK. ${ }^{27} 28$

\section{Model development}

We used SD modelling to model women's progression through different stages of ANC pathways and their decision-making processes when seeking ANC and delivery services, and to quantify neonatal mortality under different health-seeking scenarios.

The model development started with a high-level problem structuration exercise including conceptualisation of health-seeking behaviour of pregnant women from pregnancy to delivery, their consequent choice of available services, important milestones during pregnancy (states) and types of ANC services available. A qualitative schematic map was constructed to describe the process of pregnant women (surrounded by members from different parts in their social network) moving to different sectors to take up ANC services at three time points (the beginning of three trimesters) and delivery services (at the end of trimester 3 ) (figure 1A).

We then constructed a qualitative mapping of causality relationships (the causal loop diagram; figure 1B) to portray the processes of pregnant women seeking ANC and delivery services from professional providers as influenced by factors such as household wealth, social mobility levels and their social networks (family members and other women in the community).

Figure 1B depicts, for example, how an increase in the level of neonatal/maternal mortality leads to greater awareness of risk in not seeking professional ANC and delivery services, increasing women's awareness of the importance of seeking ANC services, in turn leading 


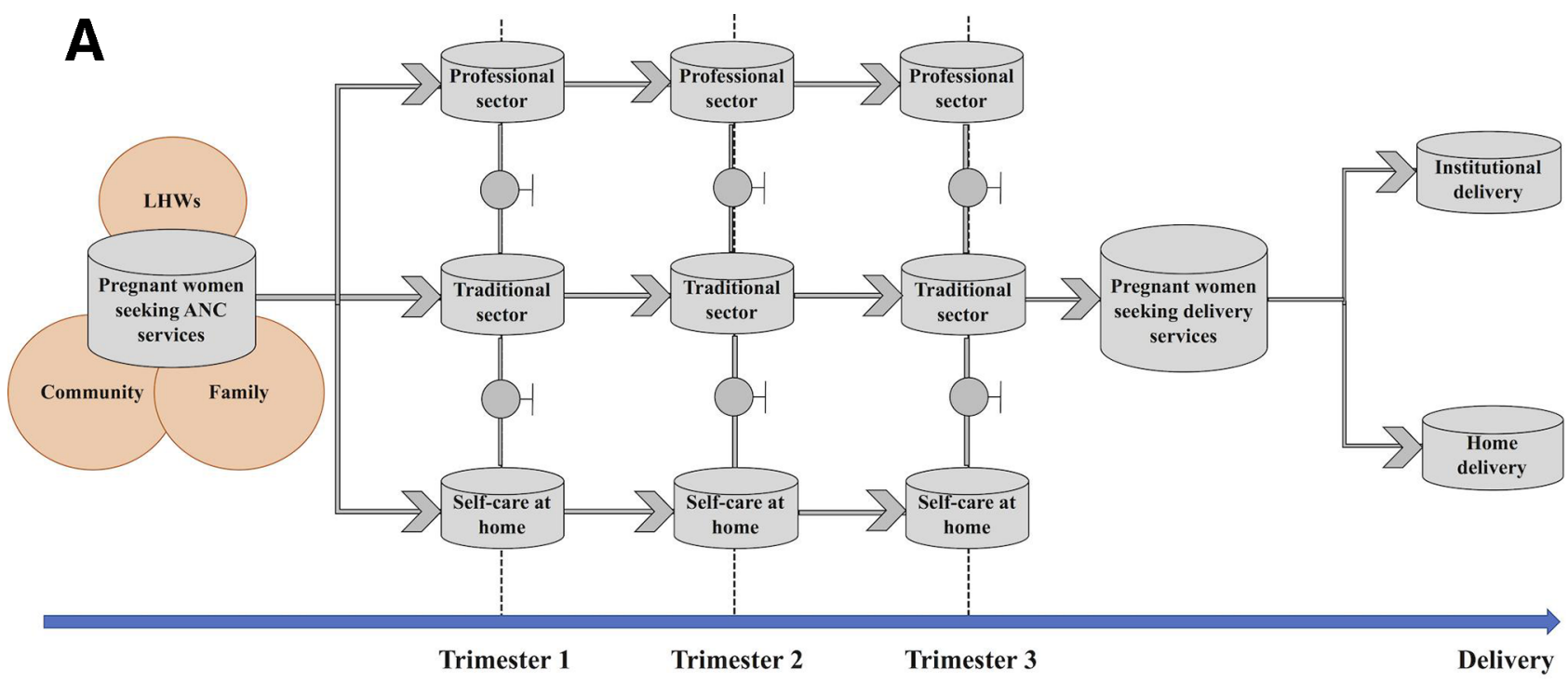

$\sum$ Patient flow with one direction marked by the arrow

Social network influence factors

Free flow, no limit of direction or frequency

Accumulation of patients

Key ANC: antenatal care LHW: Lady Health Worker

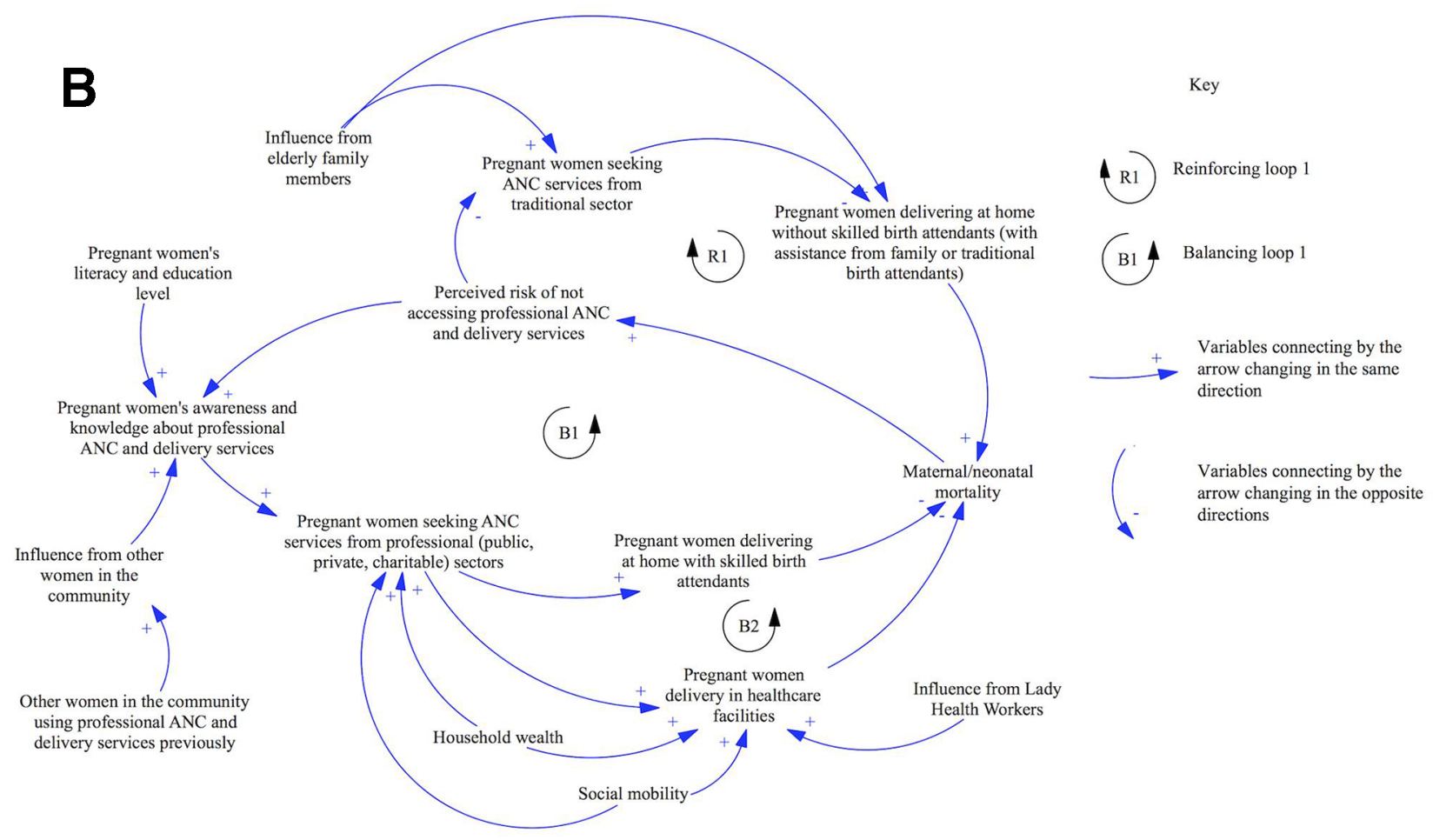

Figure 1 (A) Conceptual map of health-seeking for pregnant women using system dynamics model. (B) Causal loop diagram for health-seeking behaviour of pregnant women.

to increased uptake from healthcare professionals to reduce neonatal and maternal mortality (closed chain interactions labelled B1). By contrast, family elders may influence women to seek ANG from traditional sector, with delivery at home and higher risk of maternal and neonatal death.

\section{Simulation model}

We used a 'Stock and Flow' methodology to develop a simulation model representing the progression of women through stages of pregnancy. Stocks represent the variable states of the system and are measured by a 'level'. In our model, stocks correspond to the number of pregnant 
women at the different stages of pregnancy (eg, conception, trimester 1 , trimester 2 , trimester 3 , delivery). Flows represent the number of women moving from one stage of pregnancy to the next per unit of time. The flow rates are expressed as mathematical equations.

A stock and flow diagram is provided in online supplementary material 1 to represent the pathway of women (based on our cohort study) who decide to access ANC provided in public healthcare facilities from conception through pregnancy, to delivery. In the stock and flow diagram, the first four stocks starting from the left of the diagram represent the number of pregnant women and the number of women seeking ANC from public healthcare facilities in trimester 1 , trimester 2 and trimester 3, respectively. After trimester 3, the pathway splits into three separate flows representing the number of women choosing the three possible delivery modes: 'unattended home delivery', 'attended home delivery' or 'institutional delivery'. The final stock to the right represents neonatal mortality for women in this pathway. The full simulation model includes all the stock and flow diagrams representing all possible pathways of pregnant women accessing ANC provided in public, private, traditional and charitable sector. The outcome measure modelled is neonatal mortality.

The input data used for our model came from three sources: (1) longitudinal study (from trimester 1 through to pregnancy outcome) of a cohort of 60 women that explored qualitatively through indepth interviews the decision-making processes of pregnant women, factors which influenced these decisions, at different stages of pregnancy and the services chosen by them (online supplementary material 2) (corresponding to the proportion of women in each stock of the model); (2) interviews with healthcare professionals, managers and policy makers in Pakistan examining the causal influences on health-seeking behaviours ${ }^{19}$; and (3) a review of academic literature, providing rates of mortality, institutional delivery, attended home delivery and unattended home delivery.

Parity and number of miscarriages have been reported in previous studies to have an influence on women's careseeking. ${ }^{29}{ }^{30}$ Our qualitative cohort study captured data about pregnancy history. No difference was observed in care-seeking between women of different parity in this cohort. The Pakistan Demographic and Health Survey 2017-2018 does not include analysis results in the published report on parity or other pregnancy history indicators (data set not yet available for further analysis).

\section{Assumptions}

Our assumptions include a static population, that is, the number of pregnant women within the system remains constant, thus excludes effects of migration and other cause of mortality. We assumed homogeneity of women within subgroups by socioeconomic status as this alone did not influence health-seeking behaviour. Abortion, miscarriage, stillbirth and multiple births are not incorporated in the model but can be in future modelling, if evidence of causation is established. For this model, within-sector (private, traditional, public, charitable) quality is assumed to be consistent. While we know there is variation in quality within and across sectors, ${ }^{631} 32$ there is insufficient data to parameterise these variables.

\section{Validation of the simulation model}

The model was subjected to a full set of validation tests. ${ }^{33}$ First, the qualitative map of the model developed from the findings of the cohort study and household survey (online supplementary material 2) was confirmed from (1) academic literature and (2) expert opinions of a working group of clinicians and healthcare researchers from members of the Women and Health Initiative at the Harvard T H Chan School of Public Health, with extensive expertise in maternity health in Pakistan and in different LMIC settings.

Second, the model structure and parameters passed the boundary-adequacy test, extreme condition test and dimensional-consistency test performed. ${ }^{33}$ The last test on the model was to check its ability to replicate past realworld observed data. The model predicted a neonatal mortality of 44.8 deaths per 1000 live births, close to the neonatal mortality of 46 deaths per 1000 live births reported by WHO in 2016. ${ }^{1}$ The model was deemed valid for evaluating alternative health-seeking scenarios.

\section{Health-seeking scenarios}

The model was simulated for 1000 women for 9 months (three trimesters). Four health-seeking scenarios were evaluated as below. The model was simulated for each scenario for the ANC period of 9 months, and the value of neonatal mortality was computed for each scenario.

- Scenario 1. ANC service by any provider. women access facility-based or home-based care in any sector (public, private, traditional and charitable) instead of self-care alone.

- Scenario 2. ANC service by skilled providers: women access ANC provided by skilled professionals in public, private or charitable sector instead of self-care at home.

- Scenario 3. Institutional delivery: women deliver in healthcare facilities instead of delivering at home.

- Scenario 4. Attended home delivery: women deliver at home with a skilled birth attendant instead of delivering at home without a skilled birth attendant.

For each health-seeking scenario, we simulated different uptake levels of $25 \%, 50 \%$ and $75 \%$ and at different stages of pregnancy (trimester 1 , trimester 2 , trimester 3 and delivery). For example, one simulated scenario corresponds to $25 \%$ of pregnant women accessing ANC provided in any sector (public, private, traditional, charitable) with equal proportions at the start of trimester 1. Simulation of uptake levels for each scenario was conducted in three phases. In phase 1 , health-seeking scenarios were simulated separately. In phase 2, combinations of health-seeking scenarios were 
simulated for synergetic effects. The parameters for all simulations are listed in online supplementary material 3. In phase 3, the effects of geographical locations and social network effects were simulated.

\section{Geographical grouping of women}

Above, the simulation assumed that pregnant women were a single homogeneous group. For a more granular representation, the population was then divided into groups based on geographical location: urban and rural. Rural and urban areas differ in structural provision of services. A previous study suggests that pregnant women's odds of delivering in a healthcare facility decreased by $3 \%$ per additional kilometre of distance from a healthcare facility. ${ }^{34}$ The average distance to the nearest healthcare facility was $8 \mathrm{~km}$ in rural areas and only $1 \mathrm{~km}$ in urban areas. Additionally, women who had restricted contact with healthcare information were most likely to be advised by the elder family members and to choose traditional ANC services. Each of the health-seeking scenarios was simulated for urban and rural women.

\section{Social network influence}

The social networks of women were identified from the qualitative interviews (family members and other women in the community). The advice from LHWs is also included under social network as the interactions occurred mainly in the women's home or community interactions, as well as at the LHWs' 'Health House', and LHWs do not perform formal ANC check-ups. From the interviews and direct observation in the cohort study, we found that the LHW interactions often occurred with other family members present. Interviews with women had revealed that of those women advised by LHWs, $18.2 \%$ opted for institutional delivery. We simulated the effect of uptake of ANC based on whose advice was followed and when that advice was received (figure 2).

\section{RESULTS}

In scenario 1, neonatal mortality declines to 44.6, compared with the baseline of 44.8 deaths per 1000 live births if $75 \%$ of women choose ANC services by any provider in trimester 1 . In scenario 2, neonatal mortality declines to 44.4 if $75 \%$ of pregnant women opt for care by skilled providers in trimester 1 . In scenario 3 , neonatal mortality falls to 41.5 if $75 \%$ of pregnant women opt for institutional delivery. In scenario 4, there is marginal change in neonatal mortality that falls to 44.1 if $75 \%$ of women opt for attended home delivery.

The largest reduction in neonatal mortality is achieved when there is uptake of ANC services from skilled providers, combined with institutional delivery. When these two scenarios are combined with $25 \%, 50 \%$ and $75 \%$ uptake, neonatal mortality is further reduced to 43.6, 42.5 and 40.9 , respectively.

Intervention effects became stronger as uptake increases. Figure 3 shows the neonatal mortality rates observed under different scenarios at different stages of pregnancy and different levels of uptake. When uptake increases, neonatal mortality increases under scenario 1 if ANC is sought in trimesters 2 and 3 , and under scenario 2 if ANC is sought in trimester 3. This finding is explained by the fact that most pregnant women seeking ANC in

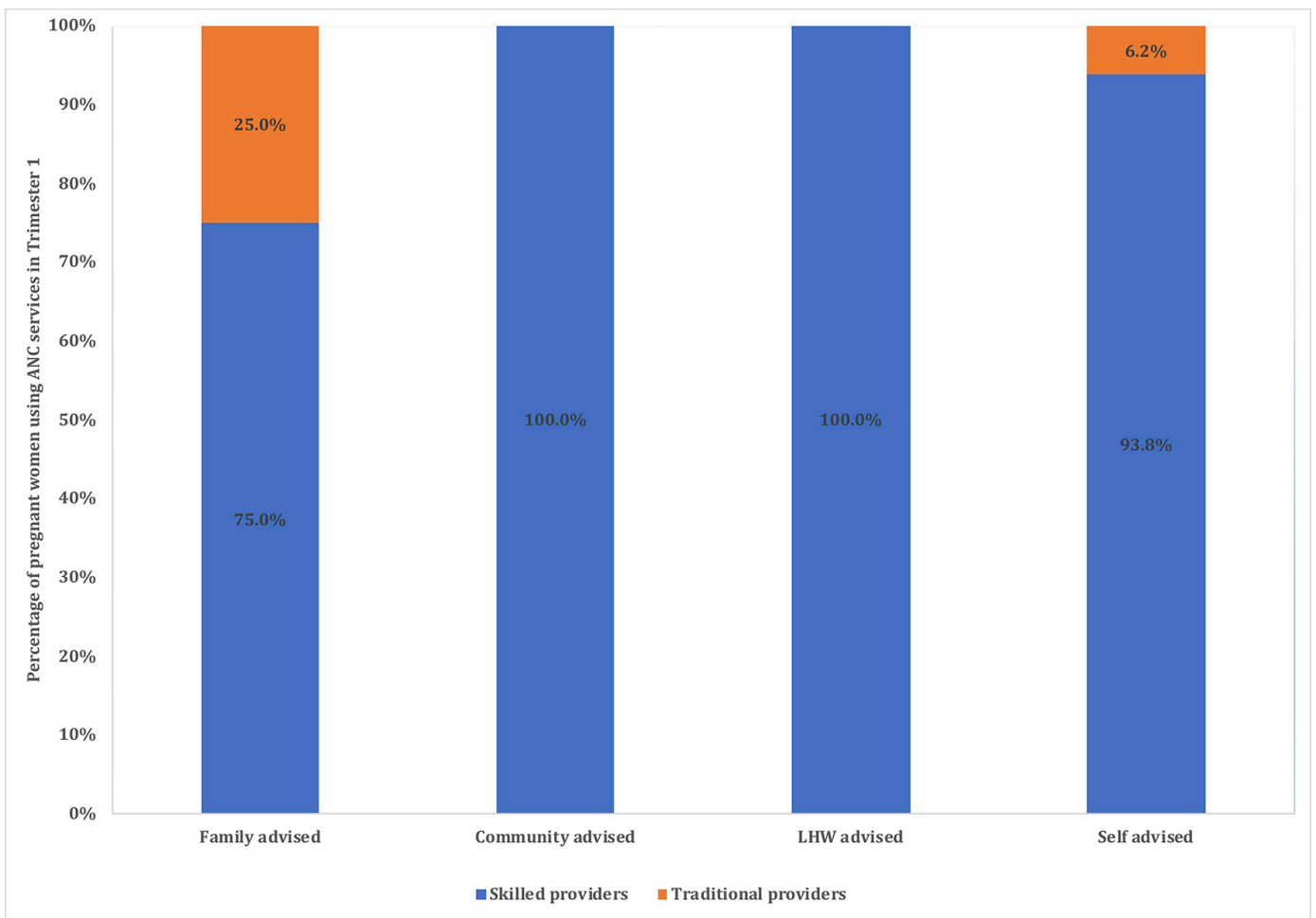

Figure 2 Antenatal care (ANC) uptake by pregnant women by type of social network advice received. 

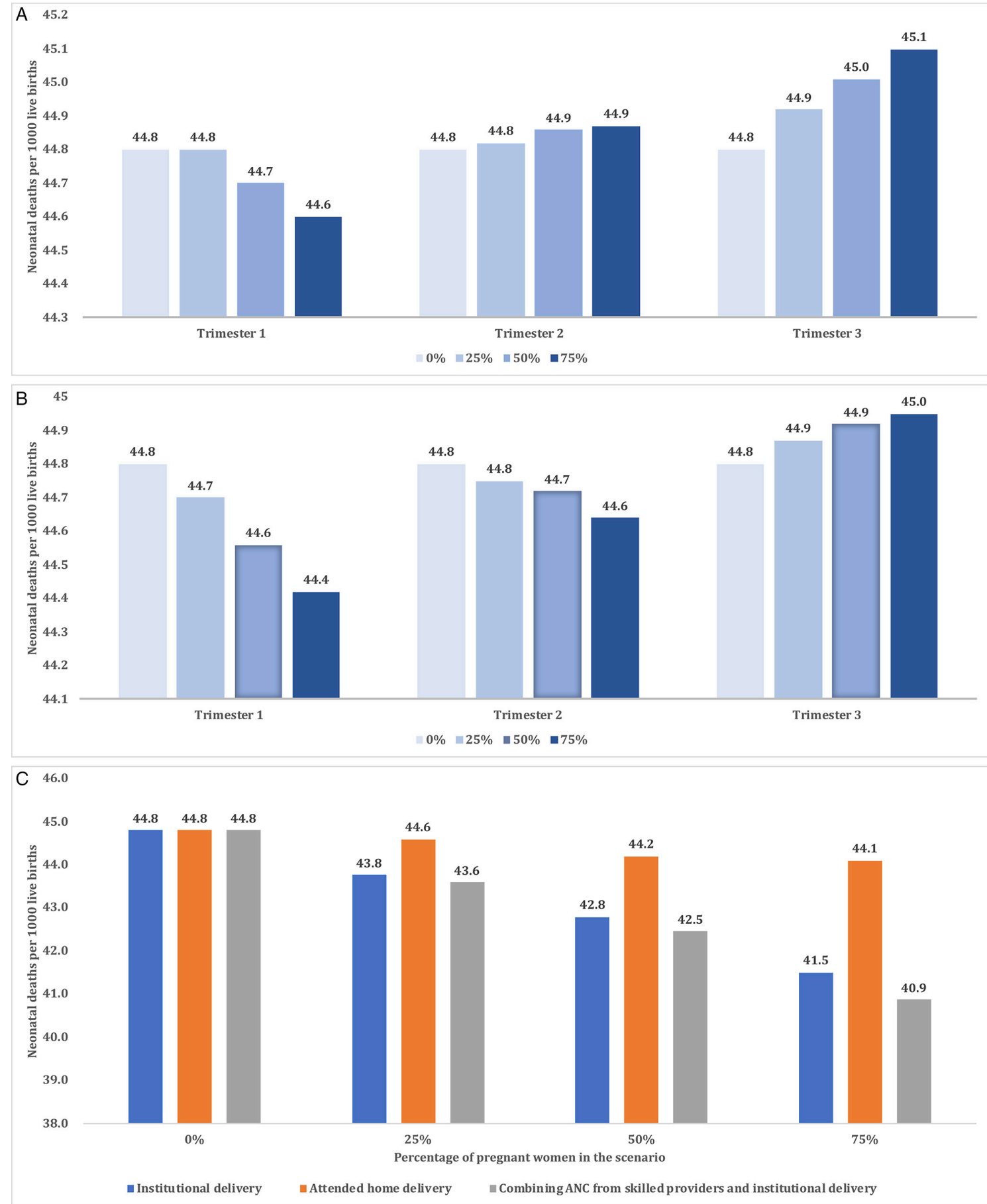

Figure 3 (A) Simulated neonatal mortality under scenario 1. (B) Simulated neonatal mortality under scenario 2. (C) Simulated neonatal mortality under scenarios 3 and 4 singularly and scenarios 2 and 3 combined. ANC, antenatal care.

trimesters 2 and 3 go for 'unattended home delivery', which is the riskiest delivery method.

\section{Geographical grouping of women}

The modified geographical simulation model was initially run under the baseline scenario and generated a neonatal mortality of 45.9 and 38.9 deaths per 1000 live births for the rural and urban subgroups, respectively. For the rural subgroup, this can be reduced to 41.1 under scenario 1 (ANC service by any provider) and 39.3 under scenario 2 (ANC service by skilled providers) if ANC is sought in trimester 1 with $75 \%$ uptake. Neonatal mortality worsens for higher uptake if ANC is sought in trimester 2 and trimester 3 under scenario 1 , and in trimester 3 under scenario 2 (figure 4 ).

For women in urban areas, the highest reduction in neonatal mortality occurs if ANC is sought in trimester 2 (34.4) under scenario 1 and in trimester 3 (35.2) under scenario 2 at $75 \%$ compliance for both scenarios. In urban areas, by contrast to rural subgroups, increased uptake even at later stages of pregnancy improves 


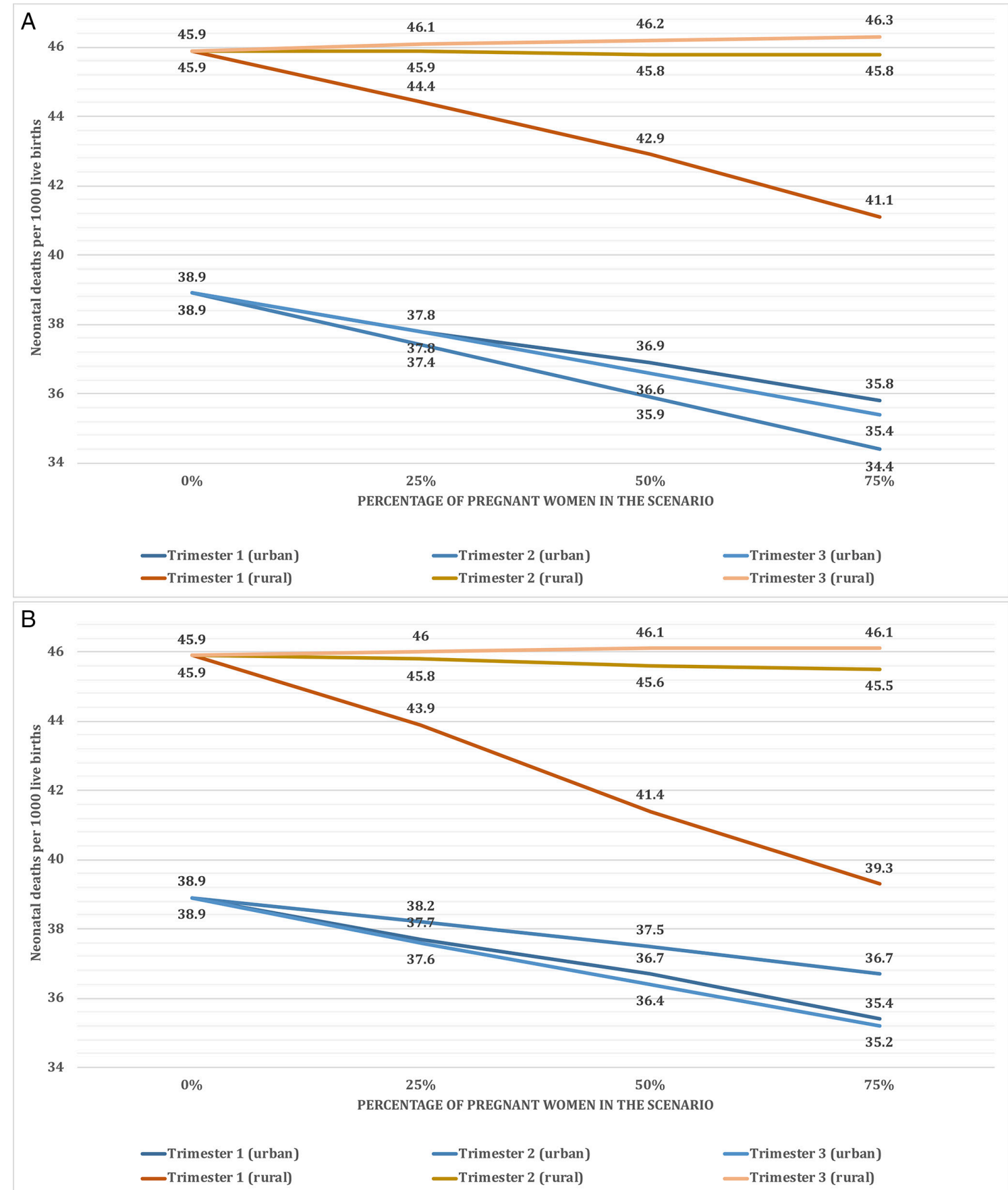

Figure 4 (A) Simulated neonatal mortality for rural and urban subgroups under scenario 1. (B) Simulated neonatal mortality for rural and urban subgroups under scenario 2. ANC, antenatal care.

neonatal mortality levels as most women seeking ANC in urban areas opt for 'institutional delivery', the safest delivery option.

For women in both urban and rural areas, the optimal intervention is similar to that of the pooled results, which involves a combination of scenario 2 (ANC by skilled professionals) and scenario 3 (institutional delivery), when neonatal mortality falls from 45.9 to 35.6 for the rural subgroup and from 38.9 to 34.2 for the urban subgroup (figure 5).

Advice by LHWs and from those in a pregnant woman's social network influences ANC health-seeking (figure 4).
For example, all women advised by LHWs at the beginning of pregnancy choose to attend ANC services from skilled providers, whereas pregnant women advised by family members, community members or self-advised switch to traditional providers or stop attending ANC services from skilled providers.

Advice from a person in a pregnant woman's social network is more influential than advice from an LHW. In trimester 2, 50.0\% of decisions to switch ANC providers (from traditional to skilled providers) were due to advice from LHWs, the remaining decisions were influenced by advice from community members, which resulted in 


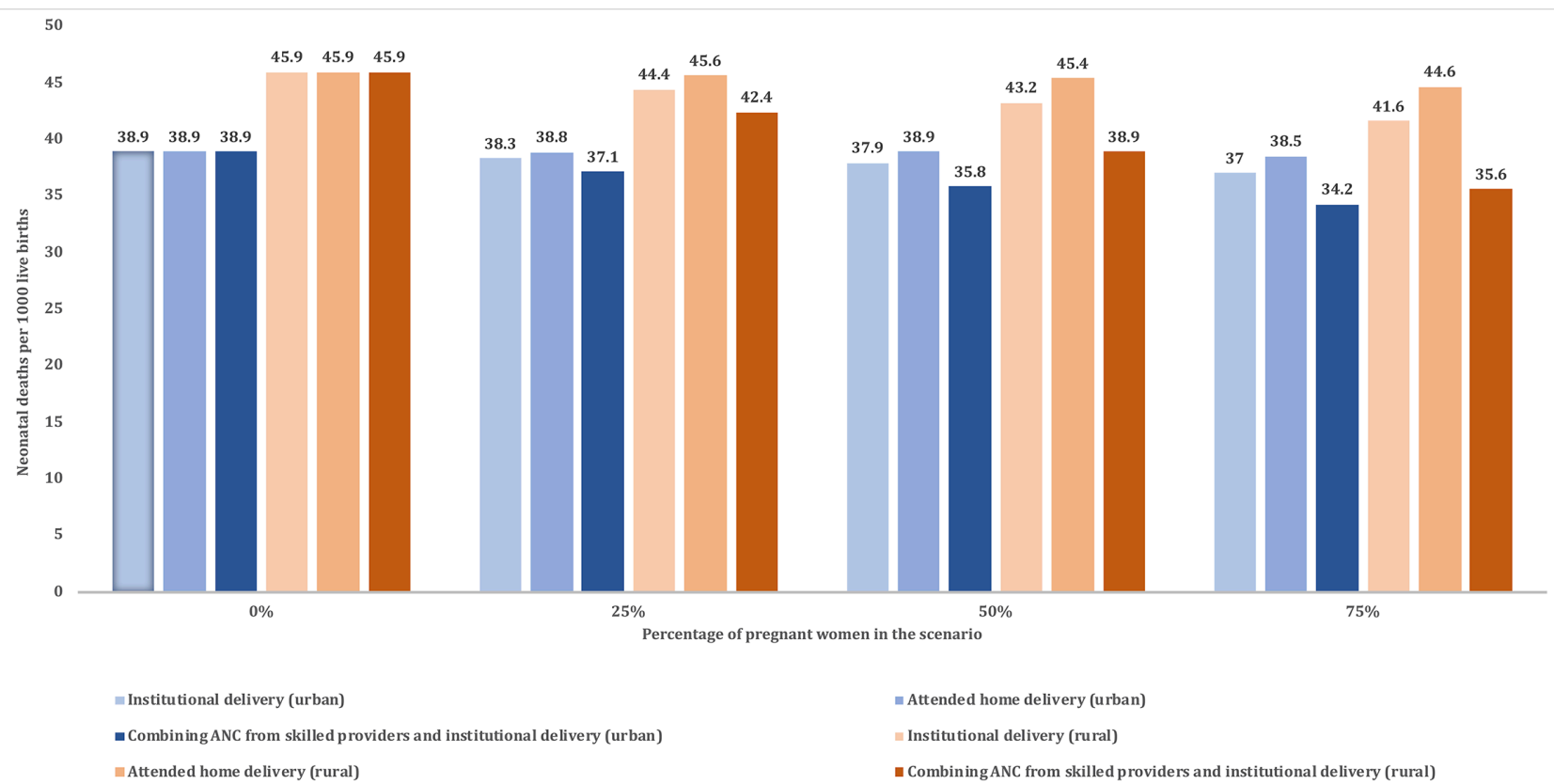

Figure 5 Simulated neonatal mortality: scenarios 3 and 4 singularly; scenarios 2 and 3 combined, rural and urban subgroups. ANC, antenatal care.

$33.4 \%$ of decisions to switch from skilled to traditional providers. In trimester 3, impact on health-seeking decision from LHWs becomes negligible. All decisions to switch are as a result of advice by family or community members, which result in $76.8 \%$ of pregnant women switching to traditional providers and only $23.2 \%$ of switching to skilled providers. The resultant neonatal mortalities following advice by different members of a pregnant woman's social network are 44.7, 44.3, 44.5 and, 45.0 for 'family-advised' subgroup, 'community-advised' subgroup, 'LHW-advised' subgroup and 'self-advised' subgroup, respectively. The 'community-advised' subgroup is the group with strong social networks and which collectively has a body of knowledge about positive practices. In addition, they had knowledge of how to access services and positive and negative experiences were directly shared.

\section{DISCUSSION}

The main findings which have implications for potential policy design in this study are that (1) interventions by LHWs after trimester 1 had a minimal impact on healthseeking behaviour and neonatal outcomes; (2) optimal benefits were achieved for urban women when ANC was accessed within trimester 2 , but for rural women within trimester 1; and (3) ANC access delayed to trimester 3 had no protective impact on neonatal mortality. Simulation results suggest that increased uptake of ANC services from skilled providers combined with institutional delivery service would result in the greatest reduction of neonatal mortality. Even the modest reduction of 4 neonatal deaths per 1000 live births is equivalent to preventing 21804 neonatal deaths per year in Pakistan. This improvement in outcomes is achievable by altering the timing of ANC uptake, within the existing healthcare resource.

The optimal time for ANC service uptake is trimester 1 during the early stages of pregnancy. If this takes place later, then contrary to what could be expected, neonatal mortality will increase as most women take up unattended home delivery. The reasons for this are complex as described in the Introduction section. When delivery free of complications was perceived by the women, most decided to deliver at home. This decision-making was influenced by social vulnerability and disempowerment, compounded by fears of the unknown and 'giving up control' in health facilities. Other responsibilities in the home, in addition to limited financial and geographical access to health facilities, impacted additionally. Decisions for home-based delivery were influenced for those women who had received ANC from skilled professionals in trimester 3, enhancing women's confidence about their pregnancy and delivery to be free of complications. Interviews with women suggested that they often interpreted a normal check-up in trimester 3 without any complications as validation of a low-risk pregnancy to proceed with a home delivery (with or without skilled attendance).

The breakdown of women by geographical location provides more insights into potential policies for reducing neonatal mortality. Late uptake of ANC by women in rural areas resulted in home deliveries and a higher neonatal mortality as service uptake increased up to $75 \%$. For women in urban areas, however, ANC uptake in later stages of pregnancy did reduce neonatal mortality because most women selected institutional delivery after ANC contact. Institutional delivery is more accessible in urban areas compared with rural areas. Interestingly, interview findings suggest 
that late health-seeking behaviours by rural women were perceived by them as 'early' intervention in preparation for delivery to 'check that everything was ok'.

The findings reinforce the critical importance of building capacity for institutional delivery especially in rural areas. Where capacity constraints are acute, ANC providers should play a more proactive role to identify women with the highest risk so that they are given priority for institutional delivery. Women delivering at home should benefit from the assistance of a skilled professional to reduce risks at birth. Previous studies have emphasised that maternal and neonatal deaths can be avoided by solving implementation issues and improving the uptake of current interventions effectively instead of developing new technologies in LMICs. ${ }^{35}$

This study has three limitations. First, the cohort study data used to parameterise the model were relatively small (60). That said, the richness and complexity captured via face-to-face indepth interviews of pregnant women through the three trimesters and after delivery would not be captured through large-scale survey methods. Second, assumptions were made about homogeneity of women within subgroups, and we also assumed for modelling purposes that the number of women in the community remained static, excluding population migration. Future modelling should include migration from rural to urban areas. Third, the model did not incorporate abortion, miscarriage, stillbirths or multiple births that might occur and their effect on outcomes.

Notwithstanding limitations, the findings have important policy implications for ANC service design in Pakistan and beyond. Interventions targeting women's health-seeking behaviour can help to improve uptake of ANC and when important preventative and diagnostic interventions can be implemented. The timing of health-seeking decisions is critical as indicated by the study, and has different implications for women in urban and rural areas and for neonatal mortality levels.

Future modelling would incorporate within-sector quality. Quality of ANC must be improved so that poor experiences do not deter women from seeking future care from public health facilities and institutional delivery. It is reported that in women whose last delivery was assisted by a skilled attendant, 10 per 1000 women reported symptoms of an obstetric fistula, ranging from 0.54 per 1000 in Burkina Faso to 32 per 1000 in Pakistan. By contrast, obstetric fistulas have been almost eliminated in high-income countries. ${ }^{36}$ Specifically, in Pakistan, only $37 \%$ of pregnant women take up ANC and one in three drop out in follow-up visits after their first ANC appointment due to 'indifferent attitude and uncertainty in availability of the staff ${ }^{37}$ Improved ANC quality would also provide LHWs with the confidence of recommending public health facilities to pregnant women. While LHWs have access to pregnant women, their influence on health-seeking behaviour of these women is suboptimal and less influential than unqualified persons in networks of pregnant women, with adverse consequences for neonatal outcomes.
The application of new research methodologies drawing on diverse disciplinary perspectives as demonstrated here has the potential for operational, implementation and health systems research relevant for decision-making at the national and international levels. ${ }^{38}$

Contributors RaA, RiA and NJZ conceived the study. RaA, RiA and NJZ composed the first draft of the manuscript. RML contributed through high-level expert input on the technical modelling process. All contributed to the model development. NJZ and RML parameterised the model. NJZ ran the validation tests. All contributed to assessment of the validation results. All contributed to revision and finalisation of the manuscript.

Funding The authors have not declared a specific grant for this research from any funding agency in the public, commercial or not-for-profit sectors.

Competing interests None declared.

Patient consent for publication Not required.

Provenance and peer review Not commissioned; externally peer reviewed.

Data sharing statement Data are available upon reasonable request.

Open access This is an open access article distributed in accordance with the Creative Commons Attribution Non Commercial (CC BY-NC 4.0) license, which permits others to distribute, remix, adapt, build upon this work non-commercially, and license their derivative works on different terms, provided the original work is properly cited, appropriate credit is given, any changes made indicated, and the use is non-commercial. See: http://creativecommons.org/licenses/by-nc/4.0/.

\section{REFERENCES}

1. World Health Organization. Maternal mortality: fact sheet, 2016 Available: http://www.who.int/mediacentre/factsheets/fs348/en/ [Accessed 04 Apr 2018].

2. Oestergaard MZ, Inoue M, Yoshida S, et al. Neonatal mortality levels for 193 countries in 2009 with trends since 1990: a systematic analysis of progress, projections, and priorities. PLoS Med 2011;8:e1001080.

3. World Bank. World development indicators. Available: https://data. worldbank.org/indicator/SP.DYN.IMRT.IN [Accessed 10 Jul 2018].

4. Who recommendations on antenatal care for a positive pregnancy experience, 2016. Available: http://apps.who.int/iris/bitstream/ handle/10665/250796/9789241549912-eng.pdf;jsessionid=644D 9E896506AAB31CEED2E88A2DBD5B? sequence=1

5. Moller A-B, Petzold M, Chou D, et al. Early antenatal care visit: a systematic analysis of regional and global levels and trends of coverage from 1990 to 2013. Lancet Glob Health 2017;5:e977-83.

6. Ahmad R, Ferlie E, Atun R. How trustworthiness is assessed in health care: a sensemaking perspective. Journal of Change Management 2013;13:159-78.

7. Zhu N, Allen E, Kearns A, et al. Lady health workers in Pakistan: improving access to health care for rural women and families. Boston, MA: Maternal Health Task Force, Women and Health Initiative, Harvard School of Public Health, 2014.

8. Lassi ZS, Middleton PF, Bhutta ZA, et al. Strategies for improving health care seeking for maternal and newborn illnesses in low- and middle-income countries: a systematic review and meta-analysis. Glob Health Action 2016;9.

9. Persson Lars Åke, Nga NT, Målqvist M, et al. Effect of facilitation of local maternal-and-newborn stakeholder groups on neonatal mortality: cluster-randomized controlled trial. PLoS Med 2013;10:e1001445.

10. Kearns AD, Caglia JM, Ten Hoope-Bender P, et al. Antenatal and postnatal care: a review of innovative models for improving availability, accessibility, acceptability and quality of services in lowresource settings. BJOG 2016;123:540-8.

11. Scammell K, Noble DJ, Rasanathan K, et al. A landscape analysis of universal health coverage for mothers and children in South Asia. BMJ Glob Health 2016;1:e000017.

12. Health System Profile, Pakistan. Regional health systems Observatory, WHO, 2007. Available: http://apps.who.int/ medicinedocs/documents/s17305e/s17305e.pdf

13. Rwashana AS, Nakubulwa S, Nakakeeto-Kijjambu M, et al. Advancing the application of systems thinking in health: understanding the dynamics of neonatal mortality in Uganda. Health Res Policy Syst 2014;12. 
14. Oxford Policy Management. Lady health worker programme evaluation 2007-09, 2010. Available: http://www.opml.co.uk/ projects/lady-health-worker-programme-evaluation-2007-09 [Accessed 04 Apr 2018].

15. Bhutta ZA, Soofi S, Cousens S, et al. Improvement of perinatal and newborn care in rural Pakistan through community-based strategies: a cluster-randomised effectiveness trial. Lancet 2011;377:403-12.

16. Sarfraz M, Tariq S, Hamid S, et al. Social and societal barriers in utilization of maternal health care services in rural punjab, Pakistan. J Ayub Med Coll Abbottabad 2015;27:843-9.

17. Zhu N. Structural and cultural influences of antenatal health seeking behaviour: system dynamics modelling. Master Dissertation. Imperial College London 2013.

18. National Institute of Population Studies - NIPS/Pakistan and ICF International. Pakistan Demographic and Health Survey 2017-18. Islamabad, Pakistan: NIPS/Pakistan and ICF International 2013.

19. Sterman JD. Learning in and about complex systems. Syst Dyn Rev 1994;10:291-330.

20. Lebcir M. Health care management: the contribution of systems thinking, 2006. Available: http://uhra.herts.ac.uk/bitstream/handle/ 2299/683/S65.pdf?sequence $=1$

21. Atun R, Menabde N. Health systems and systems thinking. health systems and the challenge of communicable diseases: experiences from Europe and Latin America. Berkshire (United Kingdom): Open University Press, McGraw Hill Education, 2008: 121-40.

22. Atun R. Health systems, systems thinking and innovation. Health Policy Plan 2012;27 Suppl 4:iv4-8.

23. Klein RW, Dittus RS, Roberts SD, et al. Simulation modeling and health-care decision making. Med Decis Making 1993;13:347-54.

24. Royston G, Dost A, Townshend J, et al. Using system dynamics to help develop and implement policies and programmes in health care in England. Syst Dyn Rev 1999;15:293-313.

25. Lane DC, Husemann E. System dynamics mapping of acute patient flows. J Oper Res Soc 2008;59:213-24.

26. Günal MM, Pidd M. Discrete event simulation for performance modelling in health care: a review of the literature. Journal of Simulation 2010;4:42-51.

27. Wakeland W, Nielsen A, Schmidt TD, et al. Modeling the impact of simulated educational interventions on the use and abuse of pharmaceutical opioids in the United States: a report on initial efforts. Health Educ Behav 2013;40(1 Suppl):74S-86.

28. Taylor K, Lane D. Simulation applied to health services: opportunities for applying the system dynamics approach. J Health Serv Res Policy 1998;3:226-32.

29. Khanam R, Creanga AA, Koffi AK, et al. Patterns and determinants of care-seeking for antepartum and intrapartum complications in rural Bangladesh: results from a cohort study. PLoS One 2016;11:e0167814.

30. Moore N, Blouin B, Razuri H, et al. Determinants of first trimester attendance at antenatal care clinics in the Amazon region of Peru: a case-control study. PLoS One 2017;12:e0171136.

31. Ahmad R. Explaining antenatal health seeking behaviour: a sensemaking perspective [PhD thesis]. Tanaka Business School, Imperial College London, 2010.

32. Pasha O, Saleem S, Ali S, et al. Maternal and newborn outcomes in Pakistan compared to other low and middle income countries in the global network's maternal newborn health Registry: an active, community-based, pregnancy surveillance mechanism. Reprod Health 2015;12(Suppl 2).

33. Sterman JD. Business dynamics: systems thinking and modeling for a complex world. Boston, MA: Irwin/McGraw-Hill, 2000.

34. Jain AK, Sathar ZA, ul Haque M. The constraints of distance and poverty on institutional deliveries in Pakistan: evidence from georeference-linked data. Stud Fam Plann 2015;46:21-39.

35. Whitworth J, Sewankambo NK, Snewin VA. Improving implementation: building research capacity in maternal, neonatal, and child health in Africa. PLoS Med 2010;7:e1000299.

36. Kruk ME, Gage AD, Arsenault C, et al. High-quality health systems in the sustainable development goals era: time for a revolution. Lancet Glob Health 2018;6:e1196-252.

37. Majrooh MA, Hasnain S, Akram J, et al. Coverage and quality of antenatal care provided at primary health care facilities in the 'Punjab' province of 'Pakistan'. PLoS One 2014;9.

38. Sheikh K, Gilson L, Agyepong IA, et al. Building the field of health policy and systems research: framing the questions. PLoS Med 2011;8:e1001073. 